\title{
The use of a novel smartphone app for monitoring male luts treatment during the COVID-19 outbreak
}

\author{
Simone Morselli ${ }^{1}{ }^{1}$ - Andrea Liaci ${ }^{1}$ - Rossella Nicoletti ${ }^{1}$ - Alessio Pecoraro ${ }^{1}$. Luca Gemma ${ }^{1}$ Paolo Polverino ${ }^{1}$. \\ Claudia Zaccaro $^{1}$ - Arcangelo Sebastianelli $\mathbb{1}^{1}{ }^{1}$ - Sergio Serni ${ }^{1} \cdot$ Pilar Laguna $^{2} \cdot$ Jean J. M. C. De La Rosette ${ }^{2}$. \\ Stavros Gravas ${ }^{3} \cdot$ Mauro Gacci $^{1}$
}

Received: 20 May 2020 / Revised: 31 May 2020 / Accepted: 2 July 2020 / Published online: 14 July 2020

(c) The Author(s), under exclusive licence to Springer Nature Limited 2020

\section{To the Editor:}

We are still experiencing the consequences from the global COVID-19 pandemic [1]. The public health emergency has resulted in a significant reallocation of health resources, with the consequent reorganization of all clinical activities in urological Departments all over the world [2].

Within the first weeks of the outbreak, Urological Departments progressively stopped the outpatient visits for benign diseases, limiting the access to the hospital exclusively for life-threatening conditions, emergency surgery, and oncological diseases [3]. Male LUTS due to Benign Prostatic Hyperplasia (BPH) were considered as a non-urgent deferrable condition; therefore, outpatient visits were postponed or canceled, even if the bothersome urinary symptoms had remarkable impact on quality of life and patient's capability to comply with the limitations imposed by the lockdown. As a result, this has led to the development of new strategies with telemedicine to appear as a pragmatic approach to screen clinically relevant cases and provide adequate treatment and follow-up [4].

An observational cohort multicenter study for treatmentnaïve patients with LUTS due to BPH is currently ongoing in five urological centers across Europe [https://clinicaltria 1s.gov/ct2/show/NCT03228485]. This trial is based on the

Mauro Gacci

maurogacci@yahoo.it

1 Department of Urological minimally invasive and Robotic Surgery and kidney Transplantation, Careggi Hospital, University of Florence, Florence, Italy

2 Department of Urology, Istanbul Medipol University, Istanbul, Turkey

3 University Hospital of Larissa, Larissa, Greece use of MyBPHCare, an app for mobile phones created by $\mathrm{Ydeal}^{\circledR}$. Aim of the present study was to assess the use of MyBPHCare App to manage males with LUTS under lockdown due to COVID-19 outbreak. For this reason, an extension of the inclusion criteria was approved by the institutional review board of a single center (Italy), to also enroll patients already on treatment for LUTS to deal with new follow-up needs in COVID-19 era.

Baseline patients' characteristics and their comorbidities are recorded in the App. Urinary symptoms, sexual function and quality of life are assessed by International Prostate Symptom Score, International Index of Erectile Function-15 and the Short Form (36) Health Survey questionnaires. Patients record their daily medication intake after a reminder generated by the App. Physicians can monitor patients' compliance to medication and contact them via the included MyBPHCare Messenger in case of low or inattentive compliance. Patients can also contact their physicians via Messenger. Information is directly stored in a data management system via an encrypted connection.

Under lockdown (March 2020-April 2020), 24 patients were enrolled in a single center. Results (Table 1) show that the enrolled patients were younger but with similar LUTSrelated characteristics compared to real-life patients [5]. Compliance reached $95 \%$ within the first 45 days. Fourteen patients (58.3\%) requested clarifications/info via Messenger. All patients were inquired after one month and expressed their satisfaction (no withdrawals).

The EAU Guidelines on Male LUTS recommend remote consultations for assessment and follow-up if capacity allows in the COVID-19 era. Symptom scores and bladder diaries can be (e)-mailed to patients [6]. However, there is a potential risk of inaccuracy and lack of relevant clinical information including the symptoms severity and the compliance to treatment that might occur during telephone consultation.

We observed a remarkable adherence to the study by patients while a significant number of patients contacted 
Table 1 Patient's characteristics and results from the use of MyBPHcare App under COVID-19, and from a series of real-life practice patients in Italy before COVID [4]. Figure on the top: MyBPHCare working concept - Hospital-Physician to House-patient communication through app.

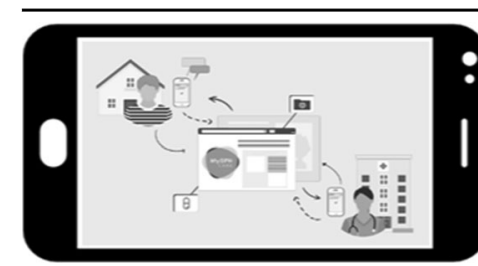

Number of patients 24

Median stay in the study (range) days

Median Age (Interquartile range) years

$\mathrm{N}$ of patients with comorbidities (\%)

Median PSA (range) $n g / m l$

Median flow rate (SD) $\mathrm{ml} / \mathrm{s}$

Median PVR (range) $m l$

Median Prostate Volume (SD) cc

Median IPSS (range)

Median IIEF-15 (range)

Median SF-36 (SD)

Physical Functioning

Role-Physical

Role-Emotional

Vitality

Mental Health

Social Functioning

Body Pain

General Health

LUTS-specific therapy

-Alpha-blockers

-5-alpha-reductase inhibitors

-Serenoa repens

Compliance ( $\%$ of daily taken medication)

Patients' withdrawals (\%)

\section{4}

45 (35-50)

60 (55-64)

$10(42.0)$

$2.2(0.9-6.2)$

$12.8(8.5-17.0)$

$90.7(0-300)$

$40(22-47)$

$11(7-15)$

$42(40-56)$

93 (85-100)

$100(50-100)$

$100(67-100)$

$60(55-75)$

64 (60-72)

65 (50-87)

78 (45-100)

65 (50-80)

\section{9}

2

4

$95 \%$

$0(0)$

14
Real life practice in Italy ${ }^{4}$
789

NA

$64.0(59-70)$

$520(65.9)$

$79.1 \%<4$

NA

51.5

45

12

NA

NA

NA

NA

NA

NA
IIEF International Index of Erectile Function, IPSS International Prostate Symptom Score, LUTS Lower Urinary Tract Symptoms, NA not applicable, PSA Prostate-Specific Antigen, PVR Post Void Residual, SD Standard Deviation, $S F-36$ Short Form (36) Health Survey. physicians for clarifications and guidance. These findings indicate that patients become immediately familiar with the app and urologists can properly follow them, according to the novel EAU 2020 Guidelines. It also confirms the great potential of telemedicine, as long as supported by validated diagnostic and therapeutic instruments, maybe even beyond the COVID-19 outbreak.

This is the very first study regarding an App proposed in the urological field, to manage a benign disorder, during the COVID-19 outbreak. Advantages include the use of selfreported validated questionnaires, scheduled reminders for taking medication and the ability to contact physicians via Messenger. Furthermore, the App is free downloadable for participants and with encrypted personal data providing full protection of privacy.
Although the sample size is small, these preliminary data are reported to provide new evidence on the feasibility of the app use under a lockdown phase. A control group and a mid-term follow up are missing: both these limitations will be overcome in the next months, with the progressive reopening of the outpatient visit and the monitoring of follow up outcomes.

In conclusion, urologists should follow-up patients with urologic benign diseases, including those with LUTS due to BPH despite the outpatient clinics lockdown during a pandemic. The App presented in this study is a useful solution to avoid contagion for both patients and physicians. Further multicenter studies are in progress, to evaluate the acceptability and use of this App for men with LUTS due to $\mathrm{BPH}$, outside a pandemic lockdown. 
Acknowledgements The study has been developed as a part of a uCARE project, supported by GSK. uCARE is the Société Internationale d'Urologie's official Office of Research.

\section{Compliance with ethical standards}

Conflict of interest The authors declare that they have no conflict of interest.

Publisher's note Springer Nature remains neutral with regard to jurisdictional claims in published maps and institutional affiliations.

\section{References}

1. COVID-19 Dashboard by the Center for Systems Science and Engineering (CSSE) at Johns Hopkins University (JHU); https://gisanddata.maps.arcgis.com/apps/opsdashboard/index. html\#/bda7594740fd40299423467b48e9ecf6.

2. Simonato A, Giannarini G, Abrate A, Bartoletti R, Crestani A, De Nunzio C, et al. Clinical pathways for urology patients during the COVID-19 pandemic. Minerva Urol Nefrol. 2020;72:376-83. https://doi.org/10.23736/S0393-2249.20.03861-8.

3. Naspro R, Da Pozzo LF. Urology in the time of corona. Nat Rev Urol. 2020;17:251-3.

4. Hollander JE, Carr BG. Virtually perfect? Telemedicine for Covid19. N Engl J Med. 2020;382:1679-81.

5. Hutchison A, Farmer R, Chapple C, Berges R, Pientka L, Teillac P, et al. Characteristics of patients presenting with LUTS/BPH in six European countries. Eur Urol. 2006;50:555-61. discussion 562.

6. Ribal MJ, Cornford P, Briganti A. European Association of Urology Guidelines Office Rapid Reaction Group: an organisation-wide collaborative effort to adapt the european association of urology guidelines recommendations to the coronavirus disease 2019 era. Eur Urol. 2020:S0302-2838(20)30324-9. 